Session 2793

\title{
Dynamic Multiple Assessment: An Instructional Method that Captures the Symbiosis of Assessment and Instruction
}

\author{
Tamara Balac, Daniel M. Gaines \\ Electrical Engineering and Computer Science Department \\ Vanderbilt University
}

\begin{abstract}
Standard instruction does not typically make effective use of assessment to improve instruction. Assessment is generally used only to assign grades to students, and no feedback is used to inform instruction. As a consequence, students may develop multiple misconceptions and receive no deep understanding of the domain. Furthermore, even when there is some assessment of the instructional methods provided by the teacher, it is separate from the curriculum. Our approach to using computers and multimedia in education addresses these issues by providing a symbiosis of assessment and instruction. We use a dynamic kind of assessment to assess the whole instructional process (not only the student, like most assessments do) and to inform instruction. We also use instruction to inform our assessment tools, hence the mutual codependence (or symbiosis) of assessment and instruction. We call the approach Dynamic Multiple Assessment (DMA) method because it dynamically assesses the student, the domain, and the instructional tools (or the teacher, indirectly). DMA adapts instruction not only to the student's level of domain knowledge, but also to their understanding of the domain, and their individual learning characteristics and preferences. DMA also gathers important information about the domain concepts, misconception harmfulness and removability, as well as the effectiveness of the instructional tools used in instruction. We implement DMA as a multimedia environment that consists of a palette of assessment and instructional tools organized to support DMA. We use multiple instructional techniques to teach concepts and promote transfer, and assess these techniques as a part of the curriculum. Furthermore, the environment serves as a powerful teacher's assistant, organizer and communicator of knowledge, as well as an interactive platform for our approach. In this paper we illustrate the DMA method by describing a suite of multimedia assessment and instructional tools designed to teach AC (Alternating Current) concepts.
\end{abstract}

\section{Introduction}

Computer technology and multimedia have the potential for increasing the quality of education. However, in order to be effective, these tools must be used appropriately. One of the major challenges is to develop instructional methods that can adapt to students' learning characteristics. This has been a limitation in many Computer-Aided Instructional (CAI) tools, other forms of computer resources (e.g. CD-ROM 
encyclopedias, Internet) as well as standard instruction. Standard instruction does not usually make effective use of assessment to improve instruction. Assessment is generally used to assign grades, and typically no feedback is used to inform instruction. As a consequence, teachers are not aware of how much knowledge their students actually possess. Students may develop multiple misconceptions and may not obtain a deep understanding of the domain.

Some of the CAI tools attempted to tailor instruction to individual's knowledge, by keeping a student model to guide the feedback and information presented ${ }^{1}$. These automatic tutors, or Intelligent Tutoring Systems (ITS), did not prove to be effective enough, and were accused of being ineffective and having "romantically unrealistic goals"2. Finally, even if ITS managed to accurately model students' knowledge, they still did not consider individuals' learning characteristics and preferences. It is how the student learns that we think is important, along with what learning strategy might work the best for them.

Our approach to using computers and multimedia in education addresses these issues by providing a symbiosis of assessment and instruction. We use a dynamic kind of assessment to assess the whole instructional process (not only the student, like most assessments do) and to inform instruction. We also use instruction to inform our assessment tools, hence the mutual codependence (or symbiosis) of assessment and instruction. We call the approach Dynamic Multiple Assessment (DMA) method because it dynamically assesses the student, the domain, and the instructional tools (or the teacher, indirectly). DMA adapts instruction not only to the student's level of domain knowledge, but also to their understanding of the domain, and their individual learning characteristics and preferences. DMA also gathers important information about the domain concepts, misconception harmfulness and removability, as well as the effectiveness of the instructional tools used in instruction. We implement DMA as a multimedia environment that consists of a palette of assessment and instructional tools organized to support DMA. We use multiple instructional techniques to teach concepts and promote transfer, and assess these techniques as a part of the curriculum. Furthermore, the environment serves as a powerful teacher's assistant, organizer and communicator of knowledge, as well as an interactive platform for our approach.

In this paper, we illustrate DMA by applying it to the domain of basic AC. We provide a brief overview of this domain followed by a description of the tools we developed to support DMA for teaching basic AC concepts. Finally, we present results from a pilot study intended to provide some early feedback on the tools.

\section{Main Components of Our Approach}

Our approach to using computers and multimedia provides a symbiosis of assessment and instruction. We use a dynamic kind of assessment to assess the whole instructional process (not only the student, like most assessments do) and to inform instruction. We also use instruction to inform our assessment tools, hence the mutual codependence (or symbiosis) of assessment and instruction. The multiple assessments we provide allow for 
personalized instruction, tailored to individual student's learning preferences and style. In the following sections, we describe our approach.

\subsection{Misconception Detection and Correction}

Some domains are more difficult to learn than others (like math and physics, for example). Such domains are usually complex and hard to understand. Consequently, these domains are generally rich in misconceptions ${ }^{3}$. Ignoring misconceptions can be harmful for future learning and understanding. Since the ultimate goal is to achieve deep understanding of the domain, we argue for emphasizing detection and correcting of misconceptions in an instruction relevant way (i.e., to inform/improve instruction).

\subsection{Symbiosis of Assessment and Instruction}

In recent years, the notion of assessment being integrated into instruction ${ }^{4}$ has been advocated by several researchers ${ }^{5}$. Assessment embedded into instruction has the ability to inform the instruction ${ }^{6}$ and can guide instructional decision making ${ }^{7}$. The instructor can use the assessment as feedback on the effectiveness of their instruction, and by establishing students' needs, find the most effective ways to meet them. This is different from traditional assessments which only purpose is grade assignment.

Assessment procedures usually concentrate on students - either to assess their knowledge, learning potential, or their needs. Nevertheless, there are three components of an instructional process - the student, the teacher and the domain (Figure 1). We see the assessment as a means of feedback not only about the student, but also about the domain of interest and the teacher as well. In the following section we talk about the specific kind of assessment procedure that we propose, which is dynamic in nature.

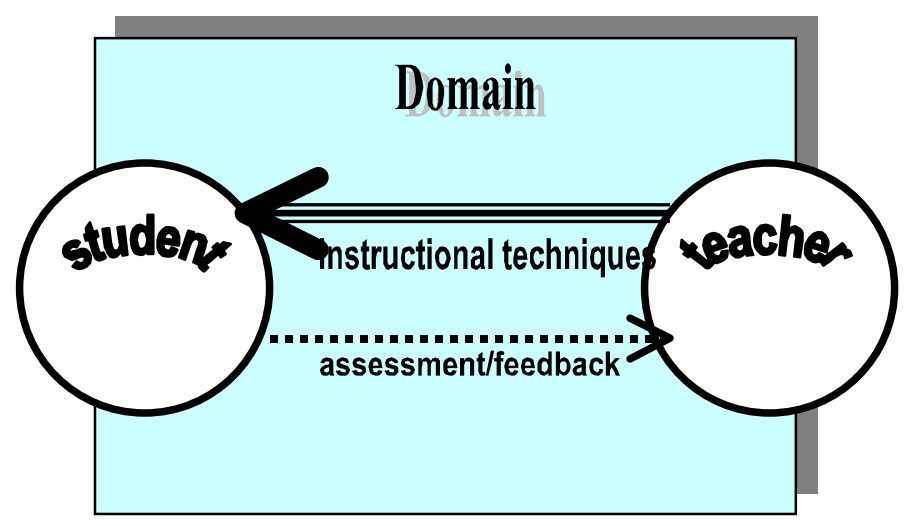

Figure 1. Instructional Process of a Domain 


\subsection{Theoretical Grounding - the Notion of Dynamic Assessment}

Dynamic assessment ${ }^{8,9}$ (DA) of learning potential originates from the work of psychologists with intellectually challenged children, but has been used to assess any individual. DA is a general concept, not a specific procedure or method. It has a general test-teach-retest form, and can be applied to virtually any kind of general or domainspecific problem. What is dynamic in DA is the nature of the interviewer's feedback they decide whether to teach a concept, probe further, back up a level to teach something more basic, or anything else their expertise guides them to do.

A DA method is based on a direct teaching intervention. Since we stress the need for having a symbiosis of assessment and instruction, using a type of assessment that incorporates instruction seems like a perfect match. A DA method, being a general dynamic concept, is also very flexible. Hence, it makes a favorable platform for implementing our idea of having multiple assessments - we can assess the student, the domain and the teacher as well.

\subsection{Dynamic Multiple Assessment}

We extend the notion of DA by implementing the Dynamic Multiple Assessment (DMA). DMA assesses all of the components of an instructional cycle: a student, the domain, and the teacher. We think that this kind of a complete assessment has the power to fully inform instruction and provide the best possible feedback for helping the student learn and understand. It does this by considering and assessing all the factors that influence instruction. DMA consists of the following assessments:

\begin{tabular}{|l|} 
Student assessment: \\
1 - assessment of individual's domain knowledge \\
2 - assessment of individual's understanding of the domain \\
3 - assessment of individual's instructional needs \\
4 - assessment of individual's learning potential \\
5 - assessment of individual's potential for transfer \\
\hline Domain assessment: \\
6 - assessment of domain learnability \\
7 - assessment of misconception harmfulness \\
8 - assessment of misconception removability \\
Teacher/instructional technique assessment: \\
9 - assessment of instructional method effectiveness \\
10 - assessment of instructional technique effectiveness to teaching a concept \\
11 - assessment of instructional technique effectiveness to teaching a concept \\
to individual
\end{tabular}

Table 1. DMA Assessments 
Assessing individual's knowledge is done by testing their current state of knowledge. It does not have to differ from standard tests. Conversely, to assess somebody's understanding, one has to find ways to make student's thinking visible, usually by carefully choosing problems that reveal students' thinking process. Assessing the understanding also incorporates testing for possible misconceptions. Assessment of an individual's instructional needs gives an insight into what kind of instruction would best help the student learn and understand. Assessment of learning potential is done in a testteach-retest manner, by comparing the gains from pre- to posttest. In our context, it is probably the least important assessment. Assessment of potential for transfer consists of questions/problems that prompt students to generalize their knowledge and use it in another situation/context. These first five types of assessment compose the student assessment part of DMA. Assessing student's domain knowledge, learning potential, and instructional needs is a part of a traditional DA method. We added the assessment of student's understanding of the domain (also part of our previous work ${ }^{6}$ ) and potential for transfer as a part of our DMA method.

Domain assessment is done by assessing the learnability of domain concepts. Some concepts are more difficult to apprehend than others are, and it is important to put more emphasis on such concepts. While detecting and correcting misconceptions in students' reasoning, the teacher also gets a sense of how harmful a misconception is for understanding and future learning, and how difficult it is to get rid of it. These assessments help the teacher create effective instructional tools. Assessment of domain learnability is a part of our previous work ${ }^{6}$, where the other two assessments are part of the DMA method.

Teacher assessment is done indirectly, by assessing the effectiveness of instructional techniques they created. Students' knowledge and understanding is also an indicator of instruction effectiveness. We can assess an instructional method in general, for example, a simulation or an analogy. We can also assess the effectiveness of a specific instructional technique to teaching a particular concept, procedure or a piece of knowledge, and moreover, to teaching it to a particular student. This helps us better determine the student's instructional needs and prescribe specific teaching methods. Teacher assessment is a new dimension of assessment that we propose.

\section{Basic AC Phenomena - An Illustrative Domain}

We chose the domain of basic AC phenomena to illustrate our methodology. Domain of electricity is a difficult domain not only to learn and understand, but to teach as well. This is due to the complexity and invisibility (i.e. one cannot "see" the current flow or "feel" the voltage) of the domain. These challenges and the abundance of misconceptions made us elect it as our illustrative domain.

Study of electricity typically starts with an introduction of the basic Direct Current (DC) concepts, like voltage, potential difference, current, resistance and power, a study of the basic components of an electric circuit, like source elements, switches, resistors, capacitors, inductors, and basic circuit laws, like Ohm's Law and Kirchhoff's Laws. 
Study of DC concepts is then followed by an introduction of Alternating Current (AC) concepts, elements and laws, and compared to the $\mathrm{DC}$ case. AC voltage and current have a temporal component added, and vary with time by periodically changing direction.

Since DC is taught before AC, knowledge of DC (or the lack of it) influences students' ability to learn and understand AC. For example, students with the "empty pipe" misconception (current flows sequentially, like water through an empty pipe, and the first bulb in a row of bulbs lights up first) have trouble accepting the fact that AC current changes direction. They are afraid that if "the electrons just stop and turn around, they may never reach the bulb". This is why our multimedia environment first checks whether a student possesses any DC misconceptions, and if so, tries to cure them. In the following section we discuss the implementation of our approach.

\section{Implementation}

To implement the eleven different kinds of assessment of DMA (Table 1), we needed various tools to test student's knowledge, teach domain concepts, re-test and test for transfer. Therefore we implemented four pools of tools: the pool of assessment, the pool of instructional, the pool of retest, and the pool of testing for transfer techniques (Figure 2).

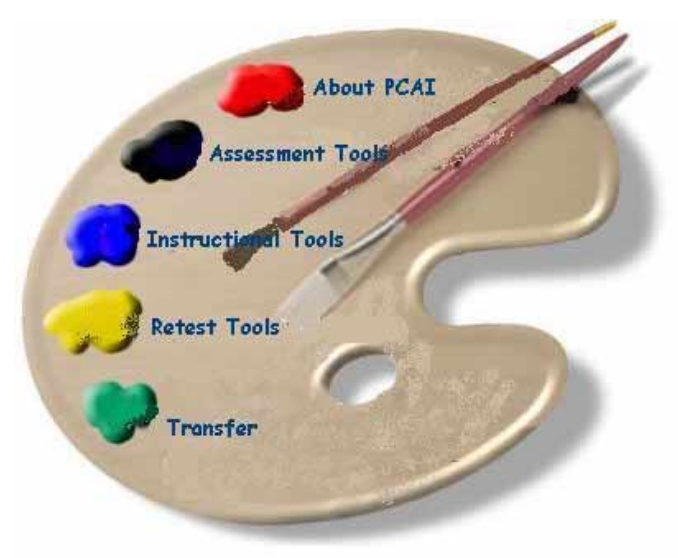

Figure 2. The four pools of DMA

The re-test tools serve to detect misconceptions and identify the level of student's knowledge and understanding. The ultimate goal is to make student's thinking visible, so that appropriate actions can be taken. The assessment pool accommodates detection of a need for introducing a new instructional tool as well. It also serves to detect harmfulness of individual misconceptions, and to assess domain concept learnability. In other words, the pool of assessment tools provides the following assessments from the Table 1: assessment 1, 2, 6, and 7. We can see two examples of assessment tools in Figure 3 and Figure 4. 


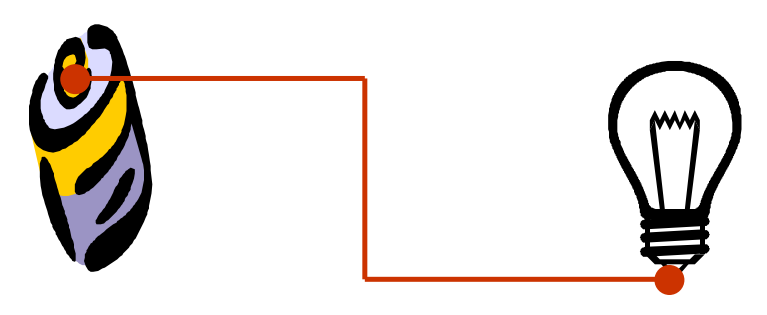

Figure 3. A DC assessment question

How does AC current flow?

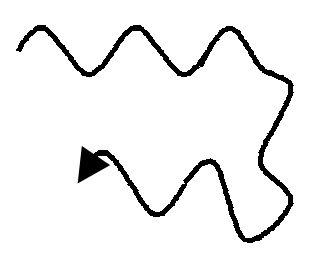

(a)

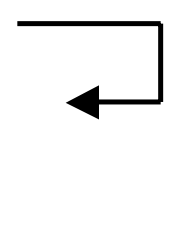

(b)

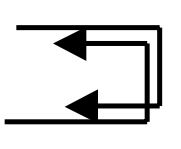

(c)

Figure 4. An AC assessment question

The pool of instructional tools accommodates DMA assessment 3, 6, 7, 8, 9, 10, and 11 from Table 1. It is divided into three categories: misconception correction techniques, teaching $\mathrm{AC}$ techniques and advanced techniques. We use multiple instructional techniques, including simulations, analogies (Figure 5), and teaching by decomposition, contradiction, learning by doing, problem solving, etc. We use humor and multimedia to keep the students interested and motivated. For example, in our "fisherman's point of view" simulation of AC current, we represent the direction of the flow of river using a little duck floating downstream (Figure 6). The ultimate goal of these techniques is to present the material in multiple ways - different points of view we hope will help different people better understand and be able to generalize their knowledge. These instructional techniques are constantly being assessed, and changed accordingly while we continue to use the environment. Some of them proved to be less effective, and were enhanced, some proved to be redundant, and were discarded. While using this pool, we also assess student's individual instructional needs, as well as the domain (i.e. concept learnability, misconception harmfulness and removability). 
Just like a heart pumps blood through our arteries and veins , a battery "pumps" current through the wires: it does not take time for blood to reach our fingers and it does not take time for current to reach a bulb, for example.

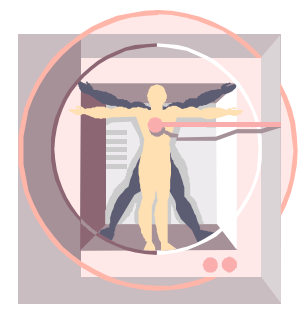

Figure 5. The heart analogy of current

And here, we have the "fisherman's" point of view of AC current - the ever-changing river, with alternating level and direction of flow, just like the changing intensity and direction of current.

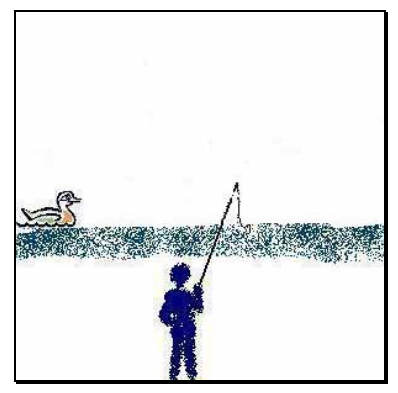

Figure 6. The "fisherman's point of view" simulation

The pool of re-test tools enables the teacher to retest student's knowledge and understanding after they received instruction. The tools try to identify any misconceptions that may not have been cured, as well as to test the new knowledge the student has been exposed to. The tools also test for learning potential (by comparing the result to the result of the testing pool), as well as assess the domain (assessment 1, 2, 4, 6, 7, and 8 from Table1). Figure 7 shows an example. 


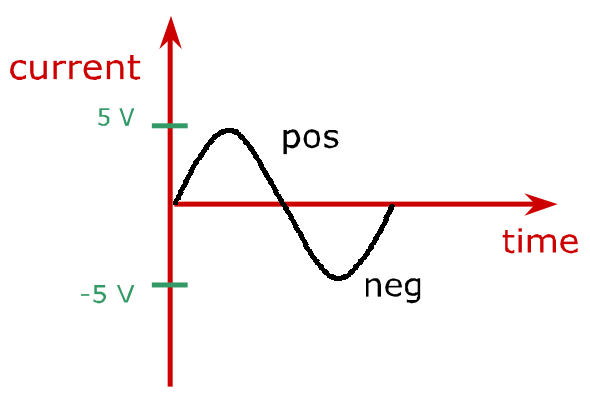

Figure 7. A DMA for AC retest question

The goal of testing for transfer techniques is to re-test the concepts in a new situation or context, and test whether students are able to generalize their knowledge. It serves as the ultimate test of deep understanding of the domain. For example, Figure 8 shows a transfer problem in which students are asked to wire a three-bedroom house in such a way that if one of the bulbs burns out, the rest of the house does not stay in the dark.

Here is a flo or plan of a three-bedroom house. Your task is to wire it in such a way that if one bulb goes out the rest of the house is not in the dark.

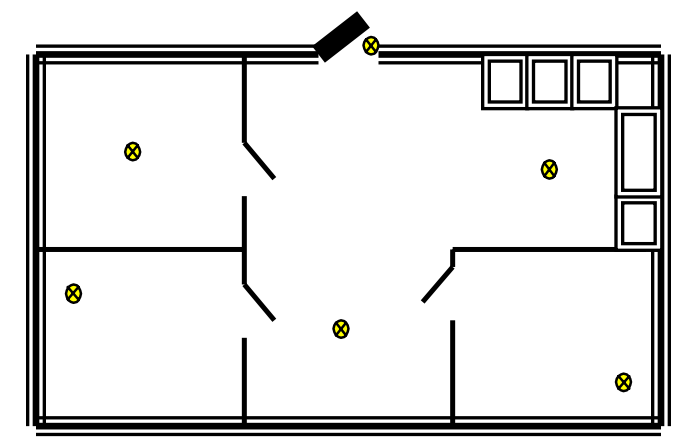

Figure 8. A DMA for AC transfer question 


\section{Pilot Study}

To evaluate our multimedia environment for teaching basic AC phenomena, we performed a pilot study with four Vanderbilt University students who completed a course in basic electronics (including DC and a the beginning of $\mathrm{AC}$ domain concepts). Each of the students went through a 30-minute session in which their knowledge and understanding of the domain were evaluated, their misconceptions were then corrected and they were taught AC current, after which they were re-tested and tested for transfer. To measure an individual's understanding of the domain concepts, we considered the number and severity of misconceptions in the pre- and post-test phase. Ideally we would like to see no misconceptions at the end of each session, but any improvement in understanding AC current would be satisfactory. Each session also gives us feedback on the instructional techniques used, and they can be improved, discarded, or new techniques introduced to accommodate the new needs just discovered. Each new technique added demands for adequate changes in the pool of assessment, retest, and transfer tools. Hence, the symbiosis of assessment and instruction provides for an ongoing enhancement of our instructional method.

The students we studied had different levels of knowledge and understanding about AC. Most of them possessed misconceptions - only one student (S1) had no common misconceptions. S1 did comment on the "fisherman's point of view" simulation (Figure 6) and another technique (in which $\mathrm{AC}$ voltage that causes $\mathrm{AC}$ current to change direction is presented as different-sized DC batteries that are flipped when voltage is negative) as being very helpful in visualizing and "getting the AC current." Other students demonstrated the "empty pipe" misconception (S2 and S4), "waving AC current" misconception (S2), and "current cannot be negative" misconception (S2, and S3). One student (S2) possessed the "clashing currents" misconception in which a negative and a positive charge flow from different battery poles and meet up in the middle to light a bulb. Another student (S3) thought that AC current does not change direction, it just "oscillates in one place."

After each of the students spent 30 minutes of one-on-one instruction with the instructor, we recorded no misconceptions in their reasoning. Our method managed to cure all of the misconceptions and students positively answered all of the new AC questions. Some students (S2, S4) had slight problems with the last transfer question in which we ask whether a sinusoid shifted up the $\mathrm{x}$-axis (so that it does not touch the negative area) represents DC or AC current.

Overall, the students liked the environment very much. Here is some of their feedback:

S2: "I thought the simulation and the little ducky were excellent. It helped me really see what is going on. I also liked the flipping batteries - it helped simplify the sinusoid."

S4: "All those different clips helped me understand the stuff better. I loved the simulation. It really helped me visualize AC current - something I was not able to do before. I wish we had stuff like this instead of all those formulas [in class]." 


\section{Conclusions}

As computers take on a larger role in the classroom, it is becoming increasingly important that we develop methods for making effective use of these valuable resources. We have proposed an approach to the design and use of computer tools which we call Dynamic Multiple Assessment (DMA). The major benefit of the DMA approach is that it captures the symbiosis of assessment and instruction. DMA assesses the whole instructional process: the student, the domain, and the instructional techniques used (as opposed to just a student, like most assessments do).

To illustrate DMA, we built a multimedia environment for teaching basic AC domain concepts. We chose the domain of AC because it is a challenging domain that is complex, invisible, rich in misconceptions, and difficult to teach and understand. We implemented the eleven assessments of the DMA method using four pools of tools: test, instructional, retest and transfer pool. Our pilot study provides encouraging feedback on our early designs. Using the methods, we were able to detect several misconceptions that students had after receiving standard instruction. During only 30 minutes, we were able to detect and correct all of the misconceptions, and accomplish a very satisfying level of knowledge and understanding of AC. Only two students had some trouble with one of the transfer questions. Even though we do not expect all students to answer all of the transfer questions correctly, a new instructional technique could be added to address this. Of course, this was a small study, used to gather early feedback on our development. In the future, we will conduct a larger study in order to perform a more thorough evaluation of the DMA approach.

Finally, our work also contributes a resolution to the dilemma of how to use all the advancements in computer technology and multimedia in education, as well as ways to provide personalized computer-aided instruction that adapts to student's individual learning characteristics, needs and preferences. This work represents a step towards our larger goal of developing a methodology to guide the development of DMA tools in other domains. The methodology will provide a set of steps and guidelines to help an instructor develop a pool of instructional, assessment, testing and re-testing tools. It will also assist in organizing the tools and scheduling their application to facilitate DMA.

\section{References}

1. Wenger, E. (1987). Artificial Intelligence and Tutoring Systems: Computational and Cognitive Approaches to the Communication of Knowledge. Los Altos, CA: Morgan Kaufman Publishers, Inc. 2. Reusser, K. (1993). Tutoring Systems and Pedagogical Theory: Representational Tools for Understanding, Planning, and Reflection in Problem Solving. In S. P. Lajoie and S. Derry (Eds.), Computer as cognitive tools. Hillsdale, NJ: LEA.

3. Osborne, R, and Freyberg, P. (1985). Roles for the science teacher. In Osborne and Freyberg (Eds.) Learning in Science. The implications of children's science, 91-99, Portsmouth, NH: Heinemann. 4. Hunt, E., and Minstrell, J. (1994). A cognitive approach to the teaching of physics. In K. McGilly (Ed.), Classroom lessons: Integrating cognitive theory and classroom practice. Campbridge, MA: Bradford. 5. Magnusson, S. J., Templin, M., \& Boyle, R. A. (1997). Dynamic science assessment: A new approach for investigating conceptual change. Journal of the Learning Sciences, 6, 91-142. 
6. Schwartz, D., Biswas, G., Bransford, J. D., Bhuva, B., Balac, T., and Brophy, S. (1998). Computer Environments for Assessment. To appear in S. P. Lajoie (Ed.) Computers as Cognitive Tools, Volume II, No More Walls: Theory Change, Paradigm Shifts and their Influence on the Use of Computers for Instructional Purposes. Mahwah, NJ: Erlbaum.

7. Minstrell, J., and Stimpson, V. (1996). A classroom environment for learning: Guiding students' reconstruction of understanding and reasoning. In L. Schauble and R. Glaser (Eds.) Innovations in Learning New Environments for Education. Hillsdale, NJ:LEA.

8. Lidz, C. S. (1987). Dynamic assessment: An interactional approach to evaluating learning potential. New York, NY: Guilford.

9. Lidz, C. S. (1991). Practitioner's Guide to Dynamic Assessment. New York, NY: The Guilford Press.

TAMARA BALAC is currently a doctoral candidate in Computer Science at Vanderbilt University. Her research interests include computer-aided instructional tools, assessment and instruction, dynamic assessment. She received her B.S. in Computer Science from University of Novi Sad, Yugoslavia, and her M.S. in Computer Science from Middle Tenn. State U., TN. Tamara is a member of AAAI, ACM, IEEE, and the Cognitive Science Society.

DANIEL M. GAINES is an Assistant Professor of Computer Science at Vanderbilt University. He received a Ph.D. in Computer Science from the University of Illinois at Urbana-Champaign in 1998. His research interests include planning, learning, robotics and CAD/CAM integration. Dr. Gaines is a member of AAAI, ACM, ASEE, ASME, and IEEE. 